\title{
Morfología y Fisiopatología del Fibrocartílago de la ATM. Revisión sistemática.
}

\section{Morphology and Pathophysiology of the TMJ Fibrocartilage. Systemic review.}

\author{
Gustavo Moncada ${ }^{1 *}$, Rodrigo Millas ${ }^{2}$, Constanza Valdés ${ }^{1}$
}

1. Facultad de Odontología, Universidad de Los Andes. Santiago, Chile.

2. Facultad de Medicina, Universidad Andrés Bello. Santiago, Chile.

* Correspondencia Autor: Gustavo Moncada | Mons. Alvaro del Portillo 12455. Las Condes. Santiago de Chile. | Código postal:7620001| Teléfono: +569 97826334 | E-Mail: gmoncada@ adsl.tie.cl

Trabajo recibido el 20/01/2020.

Aprobado para su publicación el 25/05/2020

\section{RESUMEN}

El objetivo del presente estudio fue efectuar una revisión actualizada de la morfología, fisiopatología y aspectos moleculares de la capa fibrocartilaginosa de las ATM en humanos. Método: se realizó búsqueda electrónica y manual con los términos MeSH "TMJ" y "fibrocartilage", sin límite de fecha o idioma. Los desenlaces definidos fueron: morfología del fibrocartílago, fisiopatología del fibrocartílago articular, modeladores moleculares del fibrocartílago y deterioro del fibrocartílago. Resultados: Se identificaron 1.071 títulos de artículos que después de las exclusiones, fueron seleccionados 16 a texto completo para la revisión. El fibrocartílago (FC) que cubre las superficies de las ATM es un tejido crítico en el establecimiento y la tasa de progresión de las alteraciones óseas degenerativas, clínicamente sus discontinuidades se asocian a patologías que provocan dolor, ruidos y limitación funcional. Conclusiones: A pesar de la diversidad metodológica, heterogeneidad de objetivos y diferentes características de los estudios incluidos en la revisión, el fibrocartílago de la ATM, como tejido avascular, determina fisiología de baja capacidad reparativa y mayor frecuencia de patología del FC en mujeres en edad fértil, asociado a receptores hormonales. La fisiopatología muestra reacciones inmunitarias que incrementan la acción de MMPs, interleucinas y FNTa, responsables de la degradación de la matriz extracelular, destrucción celular y morfológica del fibrocartílago, que conducen a sintomatología inflamatoria y degenerativa de pacientes que presentan artralgias y sinovitis en las ATM.

PALABRAS CLAVE:

ATM; Fibrocartílago ATM; Alteraciones degenerativas ATM; Modulación hormonal.

Int. J. Inter. Dent Vol. 13(3); 151-156, 2020.

\section{ABSTRACT}

The aim of the present study was to carry out an updated review of the morphology, physiopathology and molecular aspects of the TMJ fibrocartilage in humans. Method; electronic/manual search was performed with the MeSH terms "TMJ" and "fibrocartilage", with no date or language limit. The defined outcomes were: morphology of the fibrocartilage, physiopathology of the articular fibrocartilage, fibrocartilage molecular modelers and fibrocartilage deterioration. Results: 1071 articles were identified and after exclusions, 16 full-texts were selected for review. The fibrocartilage (FC) that covers the surfaces of the TMJ is a critical tissue in the establishment and progression rate of degenerative bone diseases; clinically, its discontinuities are associated with pain, noise and functional limitation. Conclusions: In spite of the methodological diversity, heterogeneity of objectives and different characteristics of the patients included in the review, TMJ fibrocartilage, as avascular tissue, determines a physiology of low reparative capacity, observed with greater frequency of $\mathrm{FC}$ damage in women of fertile age, associated with a greater amount of hormonal receptors. The physiopathology shows that the immune reactions increase some MMPs, interleukins and FNTa, which are linked to the degradation of the extracellular matrix, FC cellular and morphological destruction and define the inflammatory and degenerative symptomatology of patients who present TMJ arthralgia's and synovitis.

\section{KEY WORDS:}

TMJ; Joint fibrocartilage; Degenerative joint disease; Hormone modulation.

Int. J. Inter. Dent Vol. 13(3); 151-156, 2020. 


\section{INTRODUCCIÓN}

La articulación ATM es una compleja articulación dado que permite movimientos simultáneos de rotación y traslación de la mandíbula, diferenciándose de otras articulaciones sinoviales porque se forma por crecimiento que se opone a la condensación esqueletal continua. Las superficies articulares se presentan cubiertas por una capa fibrocartilaginosa más que por un cartílago hialino y finalmente esta articulación interpone la estructura discal para que ambas superficies no contacten directamente ${ }^{(1-5)}$.

Las ATM son afectadas por patologías funcionales agrupadas en Trastornos Temporo Mandibulares (TTM) que comprenden un grupo de condiciones músculo-esqueléticas y neuromusculares que incluyen las ATM, los músculos masticadores y/o estructuras asociadas( ${ }^{(6)}$. La etiología de los TTM es multifactorial, relacionándose con aspectos funcionales, estructurales y psicológicos del paciente. La mayor prevalencia de TTM se observa en el segmento etario 18 y 45 años y su prevalencia varía significativamente debido a diferencias en la terminología descriptiva. Se estima que la prevalencia de TTM en la población general oscila entre $40 \%$ a $60 \%$ y sólo el 3,6 a $7 \%$ requieren tratamiento ${ }^{(6)}$. Los TTM afectan preferentemente al adulto joven, género femenino, sin embargo también han sido reportados en niños y adolescentes ${ }^{(6,7)}$.

Los desarreglos internos (DI) y alteraciones óseas degenerativas (AOD) son las patologías intracapsulares de mayor frecuencia y se visualizan en exámenes complementarios de Resonancia Magnética y Tomografía Computada. El colapso del fibrocartílago (FC) es la primera manifestación del sobrepaso de la reparación tisular, la enfermedad es considerada irreversible y puede conducir a severos cambios morfológicos y limitaciones funcionales de las ATM $^{(3,8-10)}$. Hechos que justifican la importancia de analizar precozmente la condición del FC, especialmente si se considera la dispersa información existente sobre el FC que cubre las superficies funcionales de la ATM y el comportamiento de los mecanismos celulares y moleculares que controlan su evolución.

El objetivo del presente estudio fue efectuar una revisión actualizada de la morfología, fisiopatología y aspectos moleculares de la capa fibrocartilaginosa de las ATM en humanos.

\section{METODOLOGÍA}

El presente estudio es parte del proyecto de investigación aprobado por el Comité Ético y Científico de la Universidad de Los Andes (CEC 201951).

Como fuentes de información se utilizó la base de datos PubMed (https://pubmed. ncbi.nlm.nih,gov) y búsqueda retrograda manual, en publicaciones sin límite de idioma ni fecha. Se utilizaron los términos MeSH "TMJ" y "fibrocartilage", solos o combinados con los términos booleanos "AND" y "OR". Los criterios de exclusión fueron manuscritos de conferencia, pacientes con cáncer o ingesta de cortico-esteroides. La selección de artículos la efectuó un investigador (GM) y las consultas, discusiones y aclaraciones se efectuaron con dos investigadores (CV, RM). Los desenlaces fueron: morfología del fibrocartílago, fisiopatología del fibrocartílago articular, modeladores moleculares del fibrocartílago y deterioro del fibrocartílago. La eliminación de artículos duplicados se efectuó por medio del programa Zotero v5.0.

\section{RESULTADOS}

Por título se identificaron 969 artículos en la búsqueda electrónica y 102 en la búsqueda manual. Después de excluir artículos por duplicados, data incompleta, aplicación de criterios de exclusión, otras condiciones músculo-esqueletales y otros tipos de estudios, se seleccionaron 16 artículos a texto completo para incluir en la revisión.

Morfológicamente, el cartílago articular es uno de los tres tipos de cartílago que se encuentran en humanos (Hialino, Fibroso y Elástico)(11-13). El cartílago articular cubre los tejidos óseos móviles en su zona epifisiaria y macroscópicamente se aprecia como estructura homogénea al corte transversal. Su espesor se relaciona con su función, encontrándose los más gruesos en rodilla $(6-7 \mathrm{~mm})$ y cadera $(2-4 \mathrm{~mm})$. Su daño se asocia con alteraciones degenerativas que progresan a los tejidos óseos, porque a pesar de ser un tejido metabólicamente activo, presenta limitada capacidad de reparación y su deterioro genera dolor articular y limitación funcional(3,11,14). En el estudio histológico de la ATM de adultos jóvenes el FC ubicado sobre el tejido óseo compacto condilar difiere de espesor según su ubicación. En la zona anterior del cóndilo varía entre 0,12 a 0,8 $\mathrm{mm}$, mientras en la zona superior varía su espesor en el rango de 0,08 a $1,0 \mathrm{~mm}$, mientras en la zona posterior se reduce al rango entre 0,08 a $0,8 \mathrm{~mm}$, medidos en el eje sagital central, mostrando gran variación individual(15,16).(Figura 1)

La fisiopatología de las lesiones del FC son descritas históricamente

\begin{tabular}{l|l}
\hline \multicolumn{1}{c|}{ Morfológicas } & \multicolumn{1}{c}{$\begin{array}{c}\text { Capas constitución } \\
\text { fibrocartílago }\end{array}$} \\
\hline - Blanco - Amarillento & $\begin{array}{l}\text { - Superficial - Fibroblastos y } \\
\text { Colágeno Tipo I (contacta con } \\
\text { - Liso y brillante }\end{array}$ \\
- Dejido sinovial) \\
- Segunda - Proliferativa \\
- Avascular & - Tercera - Condrocitos, matriz \\
- Alinfatico & cartilaginosa y fibras \\
- Espesor entre 0,08mm a 1mm & colágenas tipo I \\
- Estructuralmente homogéneo & - Cuarta - tejido conectivo \\
- Cubriendo superficies & fibroso y cartílago calcificado \\
articulares funcionales & - Las fibras colágenas que \\
firmemente adherido & atraviesan el FC se insertan \\
& en el tejido óseo \\
\hline
\end{tabular}

Figura 1. Características generales fibrocartílago articular

como de difícil solución, se ha aceptado que el FC ulcerado y destruido no se repara. Sin embargo, durante el último tiempo se ha observado que presenta capacidad para formar tejido fibroso y fibrocartilaginoso y que su función mecánica no se recupera espontáneamente después de una lesión ${ }^{(17,18)}$. En articulaciones más estudiadas como la rodilla, la reparación del cartílago articular se realiza formándose colágeno inadecuado para esa zona (Tipo I) con bajo contenido de glucoproteoglicanos y anormal distribución de las células, generando cartílago de propiedades mecánicas inferiores al original( ${ }^{(19,20)}$. Se reconocen diversos mecanismos capaces de provocar alteraciones de las propiedades histoquímicas y por lo tanto mecánicas del cartílago articular, tales como inmovilizaciones, compresiones, sobrecargas ${ }^{(17,21)}$, traumatismos directos 0 indirectos, alteraciones en la estabilidad ortopédica de las articulaciones o la influencia genética, reconociendo el trauma asociado al bruxismo, como el de mayor frecuencia en la ATM(22).

El desarrollo y mantención del FC supone activa secreción de glicosaminoglicanos (GAGs) y colágeno por los condrocitos resultando una matriz extracelular organizada (MEC) que ofrece propiedades mecánicas y fisiológicas especializadas para la recepción de cargas en la superficie ósea ${ }^{(23)}$. (Figura 2)

- Mantención basada en producción de GAG y colágeno

- Tejido de baja capacidad reparativa

- Repara formando colágeno I con bajo contenido de GAG

- Condrocito alterado causa pérdida de macromoléculas tisulares, comprometiendo la función

- Equilibrio entre síntesis y degradación se pierde por acción proteinasas

- Mayor patología FC en sexo femenino en edad fértil, asociado a receptores hormonas reproductivas femeninas

Figura 2. Características fisiopatológicas del FC articular

Moléculas remodeladoras del $\mathrm{FC}$, como relaxina $\mathrm{H} 2$, (principal forma almacenada y plasmática de relaxina en humanos), polipéptido perteneciente estructuralmente a la familia de la insulina, posee efecto a largo plazo en la composición del tejido conectivo, alterando la concentración y organización de las macromoléculas claves de la matriz a través de la modulación de su síntesis o aumentando su degradación al potenciar la expresión de las MMPs o ambas. En el fibrocartílago de la ATM se ha demostrado que la relaxina induce a la MMP-1 (colagenasa 1), 3 (estromelisina-1) y 9 (gelatinasa B) que se relacionan con pérdida de colágeno y proteoglicanos de la MEC de los tejidos ${ }^{(24,25)}$.

Hallazgos de laboratorio muestran que relaxina degrada tejidos no reproductivos como cerebro, riñón, pulmón, articulaciones sinoviales y tejido fibrocartilaginoso, aportando evidencia de la relación entre relaxina, MMP y degradación de la MEC, hechos que sugieren que la relaxina predispone a enfermedades músculo-esqueléticas en las articulaciones fibrocartilaginosas de mujeres y que presentan desórdenes de la ATM, en proporción mujer/hombre 2:1 a 6:1(26). (Figura 3)

La degradación de la cubierta de tejido conectivo fibroso del FC articular en la etapa inicial de las alteraciones degenerativas de la ATM se expresa como reducción del contenido de proteoglicanos (responsables de la retención de agua), con mantención de la lubricación y las propiedades protectoras del cartílago. Existiendo correlación entre la síntesis de 
- Moduladores síntesis MEC: Estrógenos - Relaxina H2, variaciones genéticas en el polimorfismo del receptor de vitamina D y nivel de receptores estrógenos

- Degradación: Acción MMP 1, 2 y 9 mediada por hormonas Relaxina $\mathrm{H} 2$

- Limitador degradación: Progesterona inhibe MMP 3 y 9 entre otras acciones.

Figura 3. Moléculas modeladoras del fibrocartílago

proteoglicanos y la severidad de la enfermedad. La destrucción de los proteoglicanos provocan gran absorción de fluido intersticial, resultando en disminución de la capacidad para resistir cargas, adicionalmente cuando la reparación no es exitosa la enfermedad continúa con la abrasión del fibrocartílago articular y continúa con la erosión de la capa ósea subyacente formándose, espículas marginales y pseudoquistes sub condrales ${ }^{(15,27)}$. (Figuras 4 y 5 )
- Disminución significativa de GAG
- Aumento de líquido y reducción de la capacidad para soportar cargas
- Hiperplasia fibrocartilaginosa como último esfuerzo reparativo previo al colapso
- Abrasión FC
- Erosión tejido óseo subyacente
- Aumento posibilidad de traumatizar tejido sinovial
- Clínicamente dolor, ruidos y limitaciones funcionales

Figura 4. Etapas del deterioro del FC

\footnotetext{
- Incremento cantidad y permeabilidad vasos sanguíneos hiperemia

- Actividad células inflamatorias (células T, monocitos, macrófagos, osteoblastos)

- Incremento citoquinas IL-1 $\beta$, IL-6, IL-8, y FNT- $\alpha$, estimulación granulocitos y monocitos, interferón gamma (IFN Y), IL-17 y estimulación receptores RANK-L

- Presencia proteasas, citoquinas, factores de crecimiento y liberación metabolitos del ácido araquidónico, PG-E2, tromboxanos, leucotrienos.

- Aumento actividad agrecanasa, incremento de monóxido de nitrógeno y de especies oxígeno reactivas

- Posteriormente presencia IL10, IL12 e IL17 involucradas patogénesis osteoartritis ATM, dominio expresión IL12 mRNA y reducción expresión IL10 mRNA

- Reducción TIMP e incremento MMPs
}

Figura 5. Manifestaciones Alteraciones óseas degenerativas

\section{DISCUSIÓN}

a. Morfología del fibrocartílago: La literatura identifica que el aumento de espesor del FC es mayoritariamente a expensas de las células del cartílago en vez de capas de tejido conjuntivo, con menor espesor al comparar las zonas laterales y mediales, no observándose diferencias de espesor al correlacionar las variables género, presencia o ausencia de soporte molar, espesor de corticales o desgaste dentario(28). Sin embargo, cubriendo el tejido óseo del cóndilo mandibular, existe una gruesa capa de tejido conectivo fibroso, que amortigua y distribuye las presiones mecánicas sobre la superficie ósea articular ${ }^{(4,15,16,29)}$

Se identifican 4 capas en el FC, la superficial, formada por fibroblastos distribuidos en densa capa avascular, de haces compactos de fibras colágenas, orientados en todas direcciones en profundidad y de forma paralela hacia la superficie. Este tejido es la continuación del periostio de recubrimiento óseo y en la zona de mayor roce los fascículos fibrosos más profundos se insertan en el fibrocartílago. La segunda capa es delgada y proliferativa, ubicándose principalmente en las zonas laterales de la superficie condilar, cerca de la cápsula y membrana sinovial. Está formada por células indiferenciadas y por lo tanto constituye un rol celular esencial para la formación y renovación de los tejidos. En casos de limitación de movimiento mandibular por tiempo prolongado se inutiliza completamente esta capa proliferativa, perdiendo la capacidad de neoformación y recuperación de los tejidos articulares. La tercera es la capa fibrocartilaginosa de espesor variable según la zona donde se observe, siendo la zona de mayor espesor en los sectores sometidos a mayor presión y más delgadas en las zonas alejadas del área de trabajo. Esta capa de fibrocartílago puede desaparecer en la periferia del cóndilo articular, especialmente en el área sin trabajo, incluyendo fascículos de colágeno que se insertan directamente en el hueso. Esta capa está formada por una matriz cartilaginosa, condrocitos en sus lagunas y gruesos manojos de colágeno I. Las fibras colágenas atraviesan el fibrocartílago en manojos que terminan en el tejido óseo, lo que proporciona gran adherencia y resistencia a los tejidos que cubren la superficie articular del cóndilo. La cuarta capa de cartílago calcificado, se encuentra estrechamente relacionada con los osteoblastos en zonas de neoformación de tejido óseo, proporcionando al FC la capacidad de remodelación del tejido óseo de la periferia condilar. Hecho especialmente notorio en las áreas sometidas a mayor carga y actividad articular. Por este motivo el límite entre tejido óseo y fibrocartílago se aprecia irregular $(30,31)$.

La relación de los tejidos blandos ubicados sobre el tejido óseo condilar en adultos jóvenes muestra que la capa de tejido conectivo fibroso siempre se mantiene en la superficie articular, incluso en la ausencia de capa fibrocartilaginosa. La presencia o ausencia de FC, más que el grosor general del tejido, se considera el marcador de mayor utilidad para reconocer su salud y funcionalidad. El espesor de esta capa funcionalmente no se relacionaría con las alteraciones de forma de la superficie articular y no se correlaciona con la edad en adultos jóvenes ${ }^{(32)}$. La reducción del espesor de los tejidos blandos en la parte anterior del cóndilo se observó con mayor frecuencia en pacientes con falta de apoyo dentario posterior (molares). Adicionalmente, el desgaste dentario no es adecuado predictor del espesor del FC y la presencia de FC grueso o delgado no es capaz de predecir las características del contorno del tejido óseo subyacente compacto o su grosor. Por lo tanto el clínico no debe suponer que la imagen radiográfica ósea representa la superficie articular condilar real de todos los tejidos articulares ${ }^{(32)}$.

Histológicamente se observa que sobre la superficie del FC de las ATM se ubican los tejidos sinoviales, asociados al revestimiento fibroso en las superficies funcionales, no existiendo frontera clara con el tejido sinovial cuya histología depende de la ubicación y de las exigencias funcionales ${ }^{(32)}$. Variaciones en el espesor de las distintas zonas del FC corresponden a variaciones del espesor del tejido conectivo y del cartílago. El tejido conectivo fibroso contribuye tanto a la variación total en el grosor del tejido al igual que el componente de cartílago, con presencia de células mesenquimáticas no diferenciadas distribuidas en islotes a través del tejido ${ }^{(33,34)}$.

En estudios histomorfométricos de cóndilos mandibulares de humanos adultos jóvenes, se ha observado que los FC de ATM femeninas presentaban mayor cantidad de células mesenquimáticas no diferenciadas en la zona proliferativa del FC, además de menor cartílago hiperplásico y menores irregularidades en la interfase cartílago-hueso, indicando que los cambios condilares son iniciados por alteraciones en el FC y que los cambios óseos son secundarios ${ }^{(35,36)}$

Fisiopatología del fibrocartílago articular: El rol de los condrocitos es crítico en el remodelado y recambio normal del fibrocartílago a través de la expresión de proteinasas que degradan tejidos, principalmente la familia de enzimas metaloproteinasas de la matriz $(\mathrm{MMP})^{(37)}$. Este recambio tisular normal es regulado por diversos agentes locales y sistémicos incluyendo péptidos y hormonas esteroidales, determinando equilibrio entre síntesis y degradación de la MEC (proteoglicanos).

Como muchas enfermedades articulares degenerativas, presentan alta frecuencia en el sexo femenino asociado al rol regulador de las hormonas sexuales involucradas en el control metabólico de estos tejidos ${ }^{(24,38)}$ y alta prevalencia en edad fértil, (18- 45 años), a diferencia de otras enfermedades degenerativas articulares que se observan principalmente en mujeres post menopáusicas ${ }^{(39)}$. Las causas de esta distribución de sexo y edad se asocian al potencial rol de las hormonas reproductivas femeninas, que se explica por los niveles plasmáticos hormonales y las diferentes concentraciones de receptores de estrógenos (ER- $\alpha$ y $\beta$ ), en los tejidos articulares de mujeres ${ }^{(40,41)}$.

Moléculares modeladoras del fibrocartílago: La hormona progesterona ha sido propuesta como inhibidora de varias $\mathrm{MMP}^{(42)}$ en los tejidos reproductivos y en el fibrocartílago de la ATM ${ }^{(43)}$. Los efectos fisiológicos de la progesterona son mediados por su interacción con receptores intracelulares específicos de progesterona (RP). Estos receptores pertenecen a una familia que incluye receptores específicos para otras hormonas esteroidales, como tiroidea, retinol y vitamina 
D. Además la progesterona es conocida por mitigar o disminuir la degradación tisular en tejidos reproductivos y no reproductivos como el FC de la ATM $^{(25,43)}$. El rol del RP en el recambio de tejidos articulares y su potencial contribución en las enfermedades articulares, aún no es completamente conocido. Adicionalmente, otra variable que desempeña rol asociado a la susceptibilidad por la enfermedad degenerativa de la ATM, son las variaciones genéticas en el polimorfismo del receptor de vitamina $D^{(44)}$

Los dos tejidos que muestran mayor modulación de MMPs y pérdida de matriz, son la ATM y la sínfisis púbica, ambos tienen en común, similar perfil de expresión de los receptores de estrógenos y relaxina y diferentes a aquellas células del menisco de la rodilla. Estos hallazgos sugieren diferentes tejidos objetivos en articulaciones específicas por inducción de MMPs mediadas por hormonas ${ }^{(45)}$.

Deterioro del fibrocartílago: La reacción inflamatoria como respuesta al traumatismo continuo de la membrana sinovial por la presencia de superficies articulares irregulares durante la función provoca combinación de signos y síntomas como dolor articular, impotencia funcional y ruidos junto con cambios imagenológicos, en pruebas bioquímicas de sangre y en la hemostasia al interior de la ATM(17).

Los DI y las alteraciones degenerativas presentan incremento de vasos capilares, hiperemia e infiltración de células inflamatorias tales como células T o monocitos/macrófagos observados en artroscopias y estudios histopatológicos sinoviales ${ }^{(46,47)}$, que se asocian con la presencia de daños en la membrana sinovial, donde las citoquinas inflamatorias secretadas por sinoviocitos al fluido sinovial liberan factores biológicos estimulados por interleucina (IL)-1beta (Inflamosoma) y factor de necrosis tumoral (FNT-a), que se vinculan con los principales síntomas dolorosos en la ATM afectada por inflamación estéril(48). Un gran número de proteasas, citoquinas, factores de crecimiento y metabolitos del ácido araquidónico, regulan la acción de los condrocitos y la génesis del trastorno inflamatorio de la ATM, donde la destrucción celular produce liberación de ácido araquidónico, fosfolípido de la membrana, que metabolizado por ciclooxigenasas, determina la presencia de prostaglandinas y tromboxanos y por la ruta de la lipooxigenasa, la presencia de leucotrienos, en la cascada inflamatoria. Las acciones de la prostaglandina E2 (PGE2) son múltiples y desempeñan rol fundamental en la inflamación, mediando la vasodilatación local, aumento de la permeabilidad de los vasos sanguíneos y sensibilizando los nocioceptores periféricos. Se ha encontrado PGE2 en el líquido sinovial de pacientes con trastornos temporomandibulares que se ha asociado con sinovitis artroscópica basándose en la hiperemia de la membrana sinovial(49).

Las citoquinas inducidas por estímulos inflamatorios estimulan la producción, liberación y/o activación de enzimas que degradan la MEC y conducen a la producción de mediadores inflamatorios como las prostaglandinas y leucotrienos ${ }^{(50)}$. Se demostró que varias citoquinas, incluyendo IL-1, IL-6 y TNF-a, aumentan en el líquido sinovial de la articulación temporomandibular en pacientes con trastornos temporomandibulares ${ }^{(50)}$. La IL-1 como citoquina proinflamatoria producida por monocitos, linfocitos $T$ y fibroblastos es parte de la familia IL-1 que consta de tres tipos de ligandos, dos agonistas proinflamatorios (IL-1a e IL-1ß) y un antagonista del receptor de IL-1 (IL-1RA). IL-1RA es una variante estructural de la IL-1 que inhibe los efectos proinflamatorios de la IL-1 al actuar como inhibidor competitivo de la unión de la IL-1. La IL-1ß se ha detectado en el líquido sinovial de la ATM en diversas afecciones inflamatorias ${ }^{(51)}$. El líquido sinovial de pacientes con TTM contiene mayor cantidad de IL-1ß que el líquido sinovial de voluntarios asintomáticos ${ }^{(52)}$

La IL-17, producida por linfocitos T-helper, también se ha relacionado con la patogenia de los TTM ${ }^{(53)}$, describiéndose en el inicio de la sinovitis y en la degradación del FC, es inhibidora de la proliferación de los condrocitos y promotora de la producción de citoquinas osteoclastogénicas, además de estimular la expresión de RANK-L (Receptor Activator for Nuclear Factor K B Ligand,) en sinoviocitos y osteoblastos ${ }^{(50,54)}$.

El Factor de Necrosis Tumoral Alfa (TNFa) estimula a células sinoviales para la producción de PE2 e induce a los fibroblastos la secreción de colagenasa que actúa como mediador de la reabsorción ósea. Vernal y cols. ${ }^{(55)}$ sugieren que solo IL1ß, IFN, TNFa junto a IL10, IL12 y IL17 se encuentran involucradas en la patogénesis de osteoartritis de la ATM. Sin embargo la respuesta inflamatoria de la ATM se caracteriza por el dominio de la expresión de IL12 mRNA y la reducción de la expresión de IL10 mRNA que se asocia con cambios degenerativos en la ATM, coincidiendo con Takaganagi que describe a TNF-a y IL-1 como potentes inductores de la síntesis y secreción de IL-6 y cuyo incremento en el líquido sinovial se encuentra en pacientes con artralgia y sinovitis de la ATM, actuando como potentes inductores de la expresión de RANK L, estimulando la osteoclastogenesis de la membrana sinovial inflamada(50,51).

Las metaloproteinasas pertenecientes a la familia de las endoproteínas producidas por los condrocitos y células sinoviales, son secretadas en forma de zimógeno (inactivas) y una vez activadas son capaces de destruir todos los componentes de la MEC. Si bien los datos sobre la especificidad o combinación de acciones de las MMP son limitados, se sabe que los inhibidores tisulares de las metaloproteinasas (TIMP), junto a desintegrinas controlan la proteólisis de la MEC por medio de inhibición directa, este hecho permite concluir que el aumento de TIMP daría como resultado la acumulación de MEC o fibrosis, mientras que la pérdida de TIMP produce mayor actividad proteolítica, como parte del proceso de renovación de la MEC ${ }^{(56)}$.

La actividad de las MMP puede ser regulada en tres niveles: en la síntesis y secreción de IL-1 que regula la síntesis de MMP por los condrocitos ${ }^{(57)}$; a nivel de activación, que es mediada por proteólisis ${ }^{(56,58)}$ y por la inactivación por los inhibidores TIMP(56,59). Más del $95 \%$ de las MMP presentes en los tejidos de una articulación sana se encuentran en su forma latente o inhibida ${ }^{(56,58)}$.

Las MMP se clasifican según su estructura y el sustrato donde actúan en estromelisina, colagenasa, gelatinasa (MMP-2, MMP-9) y otras ${ }^{(37)}$. En el grupo de las gelatinasas se encuentra la MMP-2 (gelatinasa A / colagenasa tipo IV) producida principalmente por fibroblastos, que degrada principalmente colágeno tipo II denaturado y colágeno tipo IV, también tiene actividad significativa sobre fibronectina, elastina, y los colágenos tipo V, VII, X y XI. La MMP-9 (gelatinasa B / colagenasa tipo V), es producida principalmente por neutrófilos, macrófagos y linfocitos. Ésta es producida en grandes cantidades por las células inflamatorias, en comparación con la MMP-2, que es producida en grandes cantidades por los fibroblastos. Por lo tanto, la expresión diferente de estas MMPs sugiere que pequeñas cantidades de MMP-2 latentes existirían esencialmente en el líquido sinovial normal y que la MMP-9 facilita la destrucción progresiva de la matriz de cartílago en la alteración ósea degenerativa(60-62). Los biomarcadores TGF $\beta 1$ y MMP, evaluados en pacientes con AOD y AOD+DD, fueron detectados en concentraciones superiores en relación a otros biomarcadores comúnmente estudiados, tales como, la IL $1 \beta$, IL 6 y TNF $\beta 1^{(60,61)}$. Las concentraciones elevadas de MMP eran esperables debido a que altos niveles de MMP, especialmente MMP3, se involucran en la degradación del cartílago, contribuyendo a la destrucción característica de la $\mathrm{AOD}^{(63)}$

Otra actividad importante es la presencia de agrecanasa, clasificada como dominio de MMPs y desintegrinas con diseño tipo trombospondina (ADAMTS), las que degradan el agrecán, que consiste en una proteína central con dos dominios globulares relacionadas estructuralmente denominados G1 y G2. Estos se separan por una región extendida conocida como dominio interglobular. La pérdida de agrecán se produce antes que la pérdida de colágeno, por lo tanto, la actividad de la agrecanasa en el líquido sinovial de la ATM podría predecir la progresión a la artritis ${ }^{(18)}$.

Desde el punto de vista morfológico y biomecánico el FC se relaciona directamente con la membrana sinovial, la que muestra en su estructura células como macrófagos tipo A que reaccionan en la enfermedad inflamatoria con sustancias anti-macrófagos aumentando los procesos inflamatorios de la membrana dado el incremento de monóxido de nitrógeno. También contiene fibroblastos tipo B derivados del linaje de los monocitos, células que presentan alta expresión de receptores de estrógenos a-inmunoreaccionales, que se relacionarían con la mayor frecuencia de enfermedades sinoviales en las articulaciones femeninas ${ }^{(64)}$.

A pesar de los grandes avances a nivel molecular, para comprender la biología de los mecanismos de las alteraciones degenerativas y el deterioro del fibrocartílago articular, los investigadores no encuentran la respuesta adecuada a estas dolencias dado que aún no se han descubierto los mecanismos íntimos, las interacciones bioquímicas y otros aspectos, que conduzcan a la prevención y/o a la recuperación de los tejidos del FC articular deteriorado, encontrándose en el grupo de patologías que requiere renovados impulsos para descubrir las fórmulas que permitan controlar los factores predisponentes y la tasa de progresión de las lesiones, en la esperanza de superar las limitaciones funcionales y el dolor de un gran número de pacientes ${ }^{(60,61,65)}$.

Como futuras fuentes de desarrollo para la prevención y reparación del FC de las articulaciones con alteraciones degenerativas, una serie de técnicas emergentes con potencial terapéutico para estos tejidos se encuentran actualmente en análisis. Entre ellas destacan el desarrollo de andamios para la reparación por medio de células propias o cultivadas, aplicación y manejo del estímulo de proteínas morfogéneticas con el objeto de favorecer la diferenciación celular, el desarrollo de nanopartículas para la aplicación local de estímulos que mejoren la adhesión celular o el desarrollo de ingeniería genética aplicada a las capas fibrocartilaginosas, que permita la reposición de células dañadas o perdidas. Estas nuevas tecnologías por separado o en conjunto son las posibles opciones que actualmente se visualizan como caminos para la aplicación del nuevo 
conocimiento al servicio de las superficies articulares dañadas en las articulaciones $^{(66,67)}$

Por este camino, nuevas técnicas estarán disponibles durante los próximos años, que podrían probar sus ventajas como técnicas mínimamente invasivas, donde se iniciará la evaluación de su eficiencia, longevidad y seguridad en estudios clínicos aleatorios de tratamiento y posteriormente de prevención de las enfermedades degenerativas que involucran al FC articular.

\section{CONCLUSIONES}

A pesar de la diversidad metodológica, heterogeneidad de objetivos y las diferentes características de los pacientes incluidos en la revisión, el fibrocartílago de la ATM, como tejido avascular, determina fisiología de baja capacidad reparativa, observándose mayor frecuencia de patología del FC en mujeres en edad fértil, asociado a mayor cantidad de receptores de estrógeno. La fisiopatología concuerda en que las reacciones inmunitarias incrementan MMPs 1,2 y 9, IL-1 $\beta$, IL-6, IL-8, FNTa, que se relacionan con la degradación de la matriz extracelular, destrucción celular y morfológica del fibrocartílago y que definen la sintomatología inflamatoria y degenerativa de los pacientes que presentan artralgias y sinovitis en las ATM.

\section{CONFLICTO DE INTERES}

Los autores declaran no presentar conflicto de interés.

\section{FINANCIAMIENTO}

Este trabajo no cuenta con financiamiento alguno.

\section{Bibliografía}

1. Wurgaft R, Montenegro MA. Desarrollo y estructura de la articulación témporomandibular. 1a ed. Santiago. 2003;15, 17, 109, 155.

2. Avery JK. Oral development and histology. Thieme, NY USA, 3rd. ed. 2001: 435 3. Roberts WE, Stocum WL. Part II: temporomandibular joint (TMJ)-regeneration, degeneration, and adaptation. Curr Osteoporos Rep. 2018;16:369-79.

4. Stocum DL, Roberts WE. Part I: Development and physiology of the temporomandibular joint. Curr Osteoporos Rep. 2018;16:360-368.

5. Ottria L, Candotto V, Guzzo F, Gargari M, Barlattani A. Temporomandibular joint and related structures: anatomical and histological aspects. J Biol Regul Homeos Agents. 2018:32(2 Suppl. 1):203-7.

6. De Leeuw R, Klasser G. Orofacial Pain: Guidelines for assessment, diagnosis and management (American Academy of Orofacial Pain). 6th ed. Quintessence Publishing Co, Inc. 2018.

7. Slade GD, Ohrbach R, Greenspan JD, Fillingim RB, Bair E, Sanders AE. Painfu temporomandibular disorder: decade of discovery from OPPERA studies. J Dent Res. 2016;95:1084-92.

8. Manfredini D, Arveda N, Guarda-Nardini L, Segù M, Collesano V. Distribution of diagnoses in a population of patients with temporomandibular disorders. Oral Surg Oral Med Oral Pathol Oral Radiol. 2012;114:e35-41.

9. Schiffman EL, Fricton JR, Haley DP, Shapiro BL. The prevalence and treatment needs of subjects with temporomandibular disorders. J Am Dent Assoc. 1990;120:295-303.

10. Nickel JC, Iwasaki LR, Y Gonzalez M, Gallo LM, Yao H. Mechanobehavior and ontogenesis of the temporomandibular joint. J Dent Res. 2018:97:1185-1192.

11. Mow VC, Colmes MH, Lai WM. Fluid transport and mechanical properties of articular cartilage: A Review. J Biomech. 1984;102:73-84

12. Ross M, Paxlina W. Histología texto y atlas color con biología celular y molecular. Tejido cartilaginoso histología. 5a. ed. Médica Panamericana: 2006;198-208.

13. O'Neill TW, Felson DT. Mechanisms of Osteoarthritis (OA) Pain. Curr Osteoporos Rep. Rep. 2018;16:611-616.

14. Sacitharan PK. Ageing and osteoarthritis. Subcell Biochem. 2019;91:123-59.

15. Buckwalter JA, Mankin HJ, Grodzinsky AJ. Articular cartilage and osteoarthritis. Instr Course Lect. 2005;54:465.480.

16. Kuettner KE, Schelerbach R, Hascall VC. Articular Cartilage Biochemistry. New York, Raven Press. 1987

17. Tanaka E, Detamore MS, Mercuri LG. Degenerative disorders of the temporomandibular joint: etiology, diagnosis, and treatment. J Dent Res. 2008;87:296-307

18. Wang XD, Zhang JN, Gan YH, Zhou YH. Current understanding of pathogenesis and treatment of TMJ osteoarthritis. J Dent Res. 2015;94:666-73.

19. Messner K. Reparación de las lesiones del cartílago articular. En: Insall J and Scott WN, Cirugía de la rodilla, Marbán: Madrid. 2004;304-40.

20. Hinton RJ, Jing Y, Jing J, Feng JQ. Roles of chondrocytes in endochondral bone formation and fracture repair. J Dent Res. 2017;96:23-30

21. Sperry MM, Ita ME, Kartha S, Zhang S, Yu YH, Winkelstein B. The interface of mechanics and nociception in joint pathophysiology: insights from the facet and temporomandibular joints. J Biomech Eng. 2017;139:0210031-02100313.

22. Castrillon EE, Exposto FG. Sleep bruxism and pain. Dent Clin North Am. 2018;62:657-63.

23. Wang MQ. Displacement and tissue remodeling of temporomandibular joint disc. Zhonghua Kou Qiang Yi Xue Za Zhi. 2017;52:143-7.

24. Kapila S, Xie Y. Targeted induction of collagenase and stromelysin by relaxin in an unprimed and $\beta$ estradiol-primed diarthrodial joint fibrocartilaginous cels but not in synoviocytes. Lab Invest. 1998;78:925-38.

25. Hashem G, Zhang Q, Hayami T, Chen J, Wang W, Kapila S. Relaxyn and beta-estradiol modulated targeted matrix degradation in specific synovial join fibrocartilages: progesterone prevents matrix loss. Arthritis Res Ther. 2006;8:R98.

26. Naqvi T, Duong TT, Hashem G, Shiga M, Zhang Q, Kapila S. Relaxin's induction of metalloproteinases is associated with the loss of collagen and glycosaminoglycans in synovial joint fibrocartilaginous explants. Arthritis Res Ther. 2005;7:R1-11.

27. Li W, Zhao S, Yang H, Zhang C, Kang Q, Deng J, et al. Potential novel prediction of TMJ-OA: MiR-140-5p regulates inflammation through Smad/TGF- $\beta$ Signaling Front Pharmacol. 2019;23:15.
28. Wetselaar P, Manfredini D, Ahlberg J, Johansson A, Aarab G, Papagianni CE, et al. Associations between tooth wear and dental sleep disorders: a narrative overview. J Oral Rehabil. 2019:46:765-75.

29. Yu D, Xu J, Liu F, Wang X, Mao Y, Zhu Z. Subchondral bone changes and the impacts on joint pain and articular cartilage degeneration in osteoarthritis. Clin Exp Rheumatol. 2016;34:929-34.

30. Greenspan JS, Blackwood HJJ. Histochemical studies of chondrocyte function in the cartilage of the mandibular condyle of the rat. J Anat 1966. 1966;100:615-26. 31. Bechtold TE, Kurio N, Nah HD, Saunders C, Billings PC, Koyama R. The roles of Indian hedgehog signaling in TMJ formation. Int J Mol Sci. 2019:20:6300.

32. Pullinger AG, Baldioceda F, Bibb CA. Relationship of TMJ articular soft tissue to underlying bone in young adult condyles. J Dent Res. 1990;69:1512-8.

33. Bibb CA, Pullinger AG, Baldioceda F. Serial variation in histological character of articular soft tissue in young human adult temporomandibular joint condyles. Arch Oral Biol. 1993;38:343-52

34. Van Bellinghen X, Idoux-Gillet Y, Pugliano M, Strub M, Bornert F, Clauss F, et al Temporomandibular joint regenerative medicine. Int J Mol Sci. 2018;19:446.

35. Solberg WK, Hansson TL, Nordström B. The temporomandibular joint in young adults at autopsy: a morphologic classification and evaluation. J Oral Rehabil. 1985;12:303-21.

36. Cui D, Li H, Xu X, Ye L, Zhou X, Zheng L, et al. Mesenchymal stem cells for cartilage regeneration of TMJ osteoarthritis. Stem Cells Int. 2017;(2017):5979741. 37. Cui N, Hu M, Khalil RA. Biochemical and biological attributes of matrix metalloproteinases. Prog Mol Biol TransI Sci. 2017:147:1-73.

38. Ahmad N, Chen S, Wang W, Kapila S. 17ß-estradiol Induces MMP-9 and MMP 13 in TMJ fibrochondrocytes via estrogen Receptor $\alpha$. J Dent Res. 2018;97:10231030.

39. Burt LA, Bhatla JL, Hanley DA, Boyd SK. Cortical porosity exhibits accelerated rate of change in peri- compared with post-menopausal women. Osteoporos Int. 2017;28:1423-1431.

40. Bueno CH, Pereira DD, Pattussi MP, Grossi PK, Grossi ML. Gender differences in temporomandibular disorders in adult populational studies: a systematic review and meta-analysis. J Oral Rehabil. 2018;45:720-9.

41. Robinson JL, Cass K, Aronson R, Choi T, Xu M, Buttenbaum R, et al. Sex differences in the estrogen-dependent regulation of temporomandibular joint remodeling in altered loading. Osteoarthritis Cartilage. 2019;25:533-543.

42. Salamonsen LA, Wolley DE. Menstruation: induction by matrix matalloproteinases and inflammatory cells. J Reprod Inmunol. 1999;44:1-27.

43. Ahmad N, Wang W, Nair R, Kapila S. Relaxin induces Matrix-Metalloproteinases- 9 and -13 via RXFP1: Induction of MMP-9 involves the PI3K, ERK, Akt and PKC- $\zeta$ Pathways. Mol Cell Endocrinol. 2012;363:46-61.

44. Yilmaz $A D$, Yazicioglu $D$, Tüzüner Öncül AM, Yilmaz E, Ereş G. Vitamin D receptor gene polymorphisms (Apa1 and Taq1) in temporomandibular joint internal derangement/osteoarthritis in a group of Turkish patients. Mol Biol Rep 2018;45:1839-48.

45. Park Y, Chen S, Ahmad N, Hayami T, Kapila S. Estrogen selectively enhances TMJ disc but not knee meniscus matrix loss. J Dent Res. 2019;98:1532-8. 46. Suzuki T, Segami N, Nishimura M, Nojima T. Co-expression of interleukin1 beta and tumor necrosis factor alpha in synovial tissues and synovial fluids of temporomandibular joint with internal derangement: comparison with histological grading of synovial inflammation. J Oral Pathol Med. 2002;31:549-57.

47. Kardel R, Ulfgren AK, Reinholt FP, Holmlund A. Inflammatory cell and cytokine patterns in patients with painful clicking and osteoarthritis in the temporomandibular joint. Int J Oral Maxillofac Surg. 2003;32:390-6.

48. Ibi M. Inflammation and temporomandibular joint derangement. Biol Pharm Bull. 2019;42:538-42

49. Martel-Pelletier J, Boileau C, Pelletier J-P, Roughley PJ. Cartilage in normal and osteoarthritis conditions. Best Pract Res Clin Rheumatolgy. 2008;22:351-84.

50. Takahashi T, Kondoh T, Fukuda M, Yamazaki Y, Toyosaki T, Suzuki R. Proinflammatory cytokines detectable in synovial fluids from patients with temporomandibular disorders. Oral Surgery, Oral Medicine, Oral Pathology, Oral Radiology, and Endodontics. 1998;85:135-41. 
51. Kellesarian SV, Al-Kheraif AA, Vohra F, Ghanem A, Malmstrom H, Romanos GE, et al. Cytokine profile in the synovial fluid of patients with temporomandibular joint disorders: A systematic review. Cytokine. 2016;77:98-106.

52. Stegenga B, de Bont LG, Boering G, van Willigen JD. Tissue responses to degenerative changes in the temporomandibular joint: a review. J Oral Maxillofac Surg. 1991;49:1079-88.

53. Fossiez F, Banchereau J, Murray R, Van Kooten C, Garrone P, Lebecque S. Interleukin-17. Int Rev Immunol. 1998;15:541-51.

54. Bonato LL, Quinelato V, Borojevic R, Vieira AR, Modesto A, Granjeiro JM, et al. Haplotypes of the RANK and OPG genes are associated with chronic arthralgia in individuals with and without temporomandibular disorders. Int J Oral Maxillofac Surg. 2017;46:1121-29.

55. Vernal R, Velasquez E, Gamonal J, Garcia-Sanz JA, Silva A, Sanz M. Expression of proinflammatory cytokines in osteoarthritis of the temporomandibular joint. Arch Oral Biol. 2008;53:910-5.

56. Arpino V, Brock M, Gill SE. The role of TIMPs in regulation of extracellular matrix proteolysis. Matrix Biol. 2015;44-46:247-54

57. Larheim TA, Westesson P, Sano T. Temporomandibular joint disk displacement: comparison in asymptomatic volunteers and patients. Radiology. 2001;218:428-32.

58. Vo NV, Hartman RA, Yurube T, Jacobs LJ, Sowa GA, Kang JD. Expression and regulation of metalloproteinases and their inhibitors in intervertebral disc aging and degeneration. Spine J. 2013;13:331-41.

59. Zhang JF, Wang GL, Zhou ZJ, Fang XQ, Chen S, Fan S. Expression of matrix metalloproteinases, tissue inhibitors of metalloproteinases, and interleukins in vertebral cartilage endplate. Orthop Surg. 2018;10:306-11.
60. Cevidanes LH, Walker D, Schilling J, Sugai J, Giannobile W, Paniagua B, et al. 3D Osteoarthritic changes in TMJ condylar morphology correlates with specific systemic and local biomarkers of disease. Osteoarthritis Cartilage. 2014;22:1657-67.

61. Ganti S, Shriram P, Ansari AS, Kapadia JM, Azad A, Dubey A. Evaluation of effect of glucosamine-chondroitin sulfate, tramadol, and sodium hyaluronic acid on expression of cytokine levels in internal derangement of temporomandibular joint. Contemp Dent Pract. 2018;19:1501-5.

62. Fang J, Xu L, Li Y, Zhao Z. Roles of TGF-beta 1 Signaling in the development of osteoarthritis. Histol Histopathol. 2016;31:1161-7.

63. Jiang Q, Qiu YT, Chen MJ, Zhang ZY, Yang C. Synovial TGF- $\beta 1$ and MMP3 levels and their correlation with the progression of temporomandibular joint osteoarthritis combined with disc displacement: A preliminary study. Biomed Rep. 2013;1:218-222.

64. Ibi M, Horie S, Kyakumoto S, Chosa N, Yoshida M, Kamo M, et al. Cell-cell interactions between monocytes/macrophages and synoviocyte-like cells promote inflammatory cell infiltration mediated by augmentation of MCP-1 production in temporomandibular joint. Biosci Rep. 2018;38:BSR20171217.

65. Damlar I, Esen E, Tatli U. Effects of glucosamine-chondroitin combination on synovial fluid IL-1 $\beta$, IL-6, TNF- $\alpha$ and PGE2 levels in internal derangements of temporomandibular joint. Med Oral Patol Oral Cirugia Bucal. 2015;20:e278-283.

66. Enea D, Cecconi S, Calcagno S, Busilacchi A, Manzotti S, Gigante A. One-step cartilage repair in the knee: collagen-covered microfracture and autologous bone marrow concentrate. A pilot study. Knee. 2015;22:30-5.

67. Ntege EH, Sunami H, Shimizu Y. Advances in Regenerative Therapy: A review of the literature and future directions. Regen Ther. 2020;20:136-53. 